\title{
Modeling of Deformation Textures Evolution in Semi-Crystalline Polymers
}

\author{
S. Ahzi , D. M. Parks and A. S. Argon \\ Department of Mechanical Engineering \\ Massachusetts Institute of Technology \\ Cambridge, MA 02319
}

\begin{abstract}
A composite model for plastic deformations of semi-crystalline polymers is used to simulate deformation textures evolution in these two-phase materials. Three types of textures are simulated: crystallographic texture, morphological texture, and molecular alignment within the amorphous domains. Predictions of these textures in deformed high density polyethylene (HDPE) are shown for a uniaxial tension test.
\end{abstract}

\section{INTRODUCTION}

The morphology of semi-crystalline polymers (Polyethylene, Polypropylene, Nylon ...) consists of crystalline lamellae and amorphous layers associated with each other in a plate-like form. In the undeformed state, these two phases are generally arranged in a spherulitic structure (Figure 1). Under plastic straining, the spherulitic morphology disappears. Due to the reorientation of the lamellae and their associated amorphous domains, a morphological texture develops under plastic deformation. The plasticity of the crystalline phase, which occurs by slip and/or twinning, gives rise to a crystallographic texture. In addition to morphological and crystallographic textures, a third type of texturing develops by molecular orientation within the amorphous phase (molecular alignment).

The aim of this work is to simulate the deformation textures (morphological, crystallographic, and molecular alignment) evolution in high density polyethylene (HDPE) by using the viscoplastic composite model of Parks and Ahzi [1]. We note that HDPE is one of the widely studied semi-crystalline polymers and its crystallinity is about $70 \%$. In this study, the plasticity of the crystalline phase is assumed to occur by slip only and the amorphous phase deformation is characterized by a non linear constitutive law accounting for the molecular alignment accompanying plastic straining. Details of the plasticity mechanisms in semi-crystalline polymers are reviewed by Bowden and Young [2]. 


\section{Formulation}

The following formulation is based on the viscoplastic composite model proposed by Parks and Ahzi [1], in which elasticity is neglected and incompressibility is assumed in both phases. In this model, we solve for the kinematics and equilibrium of a two-phase composite inclusion embedded in an infinite matrix. This composite inclusion is the basic element of semicrystalline polymers, it consists of a crystalline lamella and its corresponding amorphous layer (Figure 1).

\subsection{Crystalline phase}

Like in metals, plasticity of the crystalline phase of polymers occurs by slip, twinning and martensitic transformation. However, all of these mechanisms leave the molecular chains inextensible. In the present work, slip is assumed to be the only mechanism for plastic deformation in the crystalline lamella. The possible slip systems within the orthorhombic unit cell of HDPE crystals are chain slip with Burgers vector in the chain direction c, and transverse slip with Burgers vector perpendicular to the chain direction. These slip systems (Table 1) comprise only four independent slip systems. The missing degree of freedom is due to chain inextensibility. We assume a power law relation between the microscopic strain rate and the resolved shear stress of each slip system. Let $\mathbf{D}^{c}$ and $\mathbf{S}^{c}$ denote the strain rate and deviatoric Cauchy stress respectively, within the crystalline lamella. Following the work of Parks and Ahzi [3], a constitutive relation for the crystalline lamella can be written as follows

$$
\mathbf{D}^{c}=\left\{\dot{\gamma}_{0} \sum_{\alpha=1}^{N} \frac{1}{g^{\alpha}}\left(\left|\frac{\mathbf{S}^{c, *} \cdot \mathbf{R}^{\alpha}}{g^{\alpha}}\right|\right)^{n-1} \mathbf{R}^{\alpha} \otimes \mathbf{R}^{\alpha}\right\}\left[\mathbf{S}^{c, *}\right],
$$

where $\mathbf{R}^{\alpha}$ is the symmetric part of the Schmid tensor for slip system $\alpha, \dot{\gamma}_{0}$ is a reference strain rate, $n$ represents the inverse rate sensitivity coefficient, and $g^{\alpha}$ is the slip system shear strength for slip system $\alpha$.

$\mathbf{S}^{c, *}=\mathbf{S}^{c}-S_{C}^{c} \mathbf{C}^{\prime}$ is a reduced stress tensor with $\mathbf{C}^{\prime}=\mathbf{c} \otimes \mathbf{c}-\frac{1}{3} \mathbf{1}$ the deviatoric part of the dyadic $\mathbf{c} \otimes \mathbf{c}$. Here $\mathbf{c}$ represents the crystallographic unit vector in the chain direction (constrained direction), 1 is the second order identity tensor, and $S_{C}^{c}$ is the deviatoric stress component in the constrained direction.

The rate of change of lattice orientation is given by the lattice spin $W^{*}=$ $\mathbf{W}^{c}-\mathbf{W}^{p}$ with $\mathbf{W}^{c}$ and $\mathbf{W}^{p}$ representing the lamella spin and the plastic spin, respectively. The rate of change of the crystallographic axes, for instance chain axis c, can be expressed as:

$$
\dot{\mathbf{c}}=\mathbf{W}^{*} \mathbf{c} .
$$


2.2 Amorphous phase

The plastic deformation within the amorphous phase can be described by the double kink model of Argon [4]. We propose a power law relation between the local shear rate and its corresponding shear stress. The textural hardening due to molecular alignment within the amorphous domain is accounted for by introducing a "back stress" tensor $\mathbf{B}^{a}$ in the flow rule (Boyce et al. [5]). Let $\mathbf{D}^{a}$ and $\mathbf{S}^{a}$ denote the strain rate and deviatoric Cauchy stress respectively, in the amorphous layer. The constitutive law can be written as follows (Parks and Ahzi [1]):

$$
\mathbf{D}^{a}=\dot{\gamma}_{0}\left(\left|\frac{\mathbf{S}^{a}-\mathbf{B}^{a}}{a \tau_{0}}\right|\right)^{n-1}\left(\frac{\mathbf{S}^{a}-\mathbf{B}^{a}}{a \tau_{0}}\right),
$$

where $\dot{\gamma}_{0}$ and $\tau_{0}$ are the reference strain rate and reference stress respectively, and $n$ is the rate exponent of the amorphous domain. These material parameters can be chosen equal to those of the crystalline lamella, with $\tau_{0}=g^{[001]}$ representing the initial shear strength on the easiest slip system (chain slip). The coefficient $a$ represents the relative softness of the amorphous domain. The principal components of $\mathbf{B}^{a}$ can be expressed as a function of the principal components of the plastic stretch tensor (Boyce et al. [5]). We note that under plastic straining, the macromolecules within the amorphous domains align in the direction of the maximum plastic stretch.

\subsection{Composite Inclusion}

Each crystalline lamella and its corresponding amorphous layer can be modeled as an infinitely extended composite inclusion with a planar crystallineamorphous interface (Figure 1). Let us denote by $\mathbf{n}^{I}$ the inclusion normal and by $\psi^{I}$ the angle between $\mathbf{n}^{I}$ and the crystallographic chain direction c. If $\mathbf{L}^{I}, \mathbf{L}^{c}$ and $\mathbf{L}^{a}$ denote the average velocity gradients of the inclusion, crystalline lamella and amorphous layer respectively, the inclusion volumeaveraging dictates:

$$
\begin{aligned}
& \mathbf{L}^{I}=f_{a} \mathbf{L}^{a}+\left(1-f_{a}\right) \mathbf{L}^{c} \\
& \mathbf{S}^{I}=f_{a} \mathbf{S}^{a}+\left(1-f_{a}\right) \mathbf{S}^{c},
\end{aligned}
$$

where $\mathbf{S}^{I}$ is the average deviatoric stress within the composite inclusion, and $f_{a}$ represents the amorphous volume fraction.

The interface compatibility conditions and incompressibility impose the continuity, across the lamella-amorphous interface, of some strain rate and spin components. The equilibrium condition across the interface imposes continuity of the stress tractions. These conditions, in conjunction with equations (4), constitute a complete set of equations that is solved for local kinematics. 


\section{2-4 Localization Law}

General plastic deformation can be accommodated by the composite inclusion when $\mathbf{n}^{I}$ and $\mathbf{c}$ are not parallel $\left(\psi^{I} \neq 0\right.$.). This is the case for initially spherulitic HDPE $\left(\psi^{I}=17^{\circ}-40^{\circ}\right)$. Under plastic straining, the angle $\psi^{I}$ may decrease for some inclusions and become close to zero. In this case, because of both incompressibility and chain inextensibility, the composite inclusion becomes quasi-constrained and a general deformation cannot be accommodated in the $\mathbf{n}^{I}$ or c directions. The applicability of the Taylor model then becomes an issue for all inclusions having a small angle between $\mathbf{n}^{I}$ and $\mathbf{c}$ (quasi-constrained inclusions). In order to solve this problem, Parks and Ahzi [1] proposed a modification (relaxation) of the Taylor model to be applied to the quasi-constrained inclusions $\left(0<\psi^{I}<\psi_{0}\right)$, where $\psi_{0}$ is a suitably small angle. If we denote by $\mathbf{D}^{I}$ and $\overline{\mathbf{D}}$ the strain rate of the inclusion $I$ and the macroscopic strain rate respectively, the localization law of Parks and Ahzi [1] can be written as follows:

$$
\begin{aligned}
& \mathbf{D}^{\mathbf{I}}=\mathcal{P}<\mathcal{P}>^{-1} \overline{\mathbf{D}} \text { for } \psi^{I}<\psi_{0}, \\
& \mathbf{D}^{\mathbf{I}}=\overline{\mathbf{D}} \quad \text { for } \psi^{I} \geq \psi_{0} \text {; }
\end{aligned}
$$

where

$$
\mathcal{P}=\mathcal{I}-\frac{3}{2}\left(1-\left(\psi^{I} / \psi_{0}\right)^{m}\right) \mathbf{N}^{\prime} \otimes \mathbf{N}^{\prime}
$$

$\mathcal{I}$ is the fourth order identity tensor and $\mathrm{N}^{\prime}=\mathbf{n}^{I} \otimes \mathrm{n}^{I}-\frac{1}{3} 1$ is a tensorial representation of the lamella normal direction. $\psi_{0}$ is a critical angle, $m$ is an arbitrary power exponent and $<>$ designates the volume average over all quasi-constrained inclusions. In the numerical applications, the values of $\psi_{0}=15^{\circ}$ and $m=5$ have been chosen. To complete the description of the velocity gradient imposed on the composite inclusion, we equate the inclusion spin $\mathbf{W}^{I}$ to the macroscopic spin, $\overline{\mathbf{W}}$ :

Using the concept of convected material coordinates, Parks and Ahzi [1] proposed a precise evolution equation for the inclusion normal, $\mathbf{n}^{I}$.

\section{Results and Conclusions}

We applied the present composite model to an initially isotropic (quasispherulitic) high density polyethylene with $f_{a}=0.3$ and $n=9$. The initial distribution of chain axes $\mathrm{c}$ is isotropic (244 orientations), and the corresponding distribution of lamellae normals $\mathbf{n}^{I}$ is also random. The initial angle between $\mathbf{c}$ and $\mathbf{n}^{I}$ is approximately $30^{\circ}$ for all orientations. The slip systems of the crystalline phase of polyethylene are summarized in Table 1 , along with their estimated initial shear resistances normalized to the reference stress, $\tau_{0}$. No crystallographic strain hardening is considered in the 
following application. A uniaxial tension test is simulated for HDPE. The predicted crystallographic texture is shown by $(200)$ and (002) pole figures in Figure 2 for $180 \%$ tensile true strain. The predicted fiber texture is in agreement with experimental observations. The morphological texture is represented by the distribution of the inclusion normals shown in Figure 3. Figure 4 shows the predicted distribution of the directions of maximum stretch within the amorphous phase (molecular alignment). Comparison of our results to experimental data are satisfactory (Parks and Ahzi [6])

\section{ACKNOWLEGMENTS}

This work was supported by DARPA U.R.I program under ONR contract No. N00014-86-K-0768. We would like to thank C. Bronkhorst for presenting this work on our behalf.

\section{REFERENCES}

[1] Parks, D. M. and Ahzi, S., ms. in preparation, 1990.

[2] Bowden, P. B. and Young, R. J., J. Mat. Sci. 9, 1974, p. 2034.

[3] Parks, D. M. and Ahzi, S., J. Mech. Phys. Solids, in press, 1990.

[4] Argon, A. S., Phil. Mag., 28, 1973, p. 839.

[5] Boyce, M. C., Parks, D. M. and Argon, A. S., Mech. Materials, 7, 1988, p. 15.

[6] Parks, D. M. and Ahzi, S., Inelastic Behavior of Composite Materials, Ed. G. J. Dvorak, in press, 1990.

\begin{tabular}{|l|c|c|}
\hline & slip system & normalized resistance \\
\hline \hline \multirow{2}{*}{ Chain slip } & $(100)[001]$ & 1. \\
& $(010)[001]$ & 1. \\
& $\{110\}[001]$ & 1. \\
\hline \multirow{2}{*}{ Transverse slip } & $(100)[010]$ & 1.1 \\
& $(010)[100]$ & 1.4 \\
& $\{110\}<110>$ & 1.8 \\
\hline
\end{tabular}

Table 1: Slip systems in polyethylene crystals. 

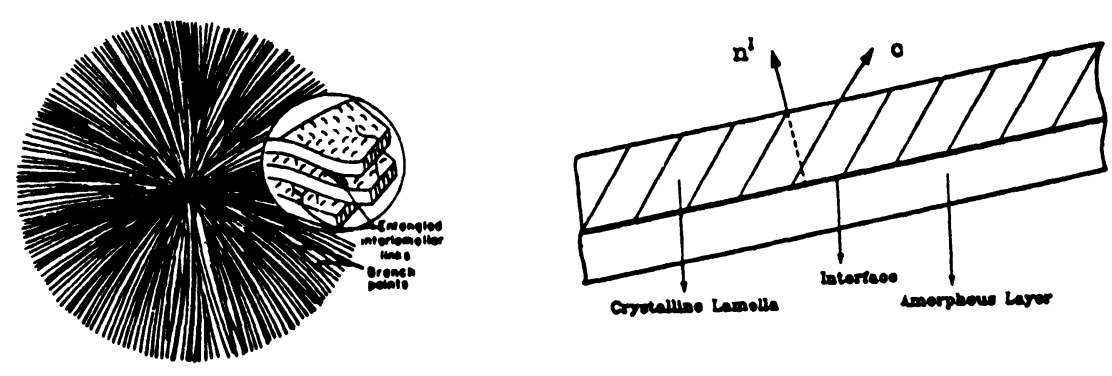

Figure 1: Schematic representation of the spherulite (a) and the composite inclusion (b).
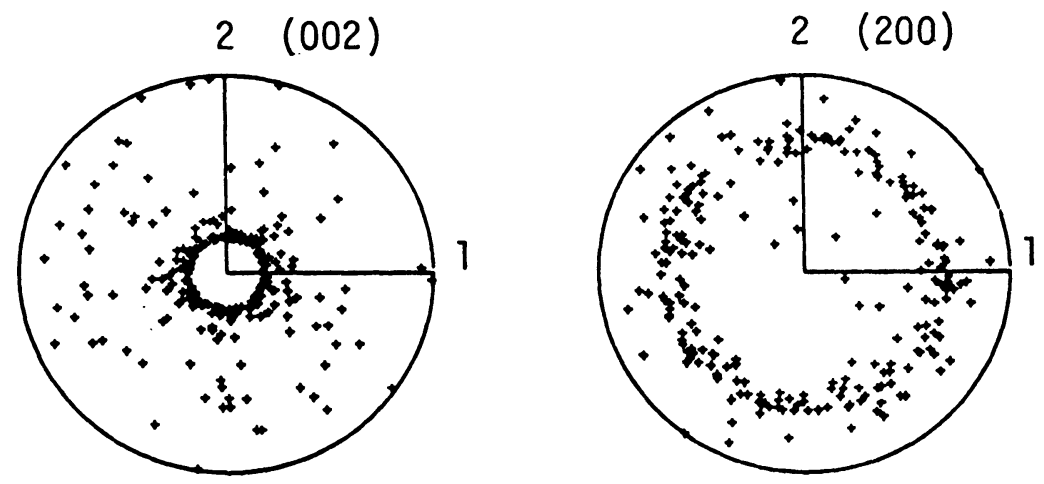

Figure 2: Crystallographic texture after $180 \%$ tension $\left(\epsilon_{e q}=1.8\right)$. Axis 3 = tensile direction.

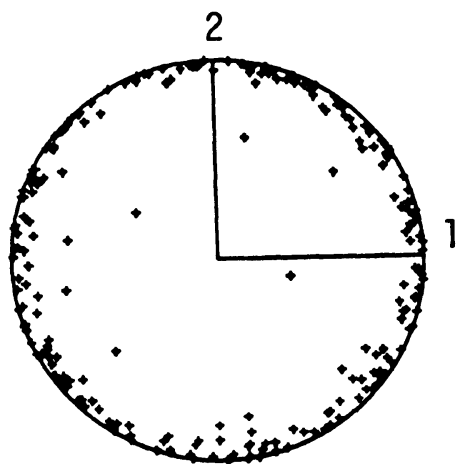

Figure 3: Inclusion normals distribution $\left(\epsilon_{e q}=1.8\right)$.

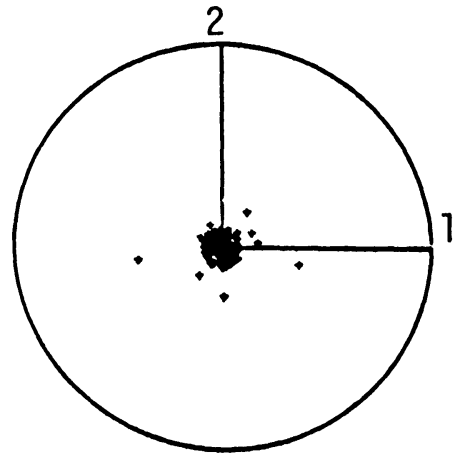

Figure 4: distribution of the directions of maximum stretch within the amorphous phase $\left(\epsilon_{e q}=1.5\right)$. 